\title{
Farbzentrenbildung in plastisch verformtem Calcit
}

\author{
W. Sterzel und E. Chorinsky \\ Institut für Anorganische Chemie der Universität Frankfurt/Main \\ (Z. Naturforsch. 23 a, 1052-1058 [1968] ; eingegangen am 13. April 1968)
}

\begin{abstract}
Farbzentren mit Absorptionen im Bereich des sichtbaren Lichtes entstehen bei Röntgen-Bestrahlung in Calcitkristallen, wenn die Kristalle durch Verformung eine hohe Störstellenkonzentration erhalten haben. Die Absorptionsspektren solcher Farbzentren werden ausgemessen und mit röntgenographisch bestimmten Störungsgraden verglichen. Die Bildung und die thermische Ausheilung der für die Entstehung von Farbzentren erforderlichen Störstellen wird quantitativ untersucht. Dabei wird festgestellt, daß mindestens zwei verschiedene Farbzentren auftreten, deren Konzentrationsverhältnis von der Temperaturvorbehandlung der gestörten Calcitproben abhängt.
\end{abstract}

Plastisch verformter Calcit färbt sich blauviolett, wenn er einer Röntgen-Bestrahlung ausgesetzt wird. Unverformter Calcit und verformte Kristallpulver der beiden metastabilen Calciumcarbonatmodifikationen Aragonit und Vaterit zeigen diese Eigenschaft nicht. Daß es sich bei der Anfärbung von Calcitpulvern oder Calcitkristallen um eine Folge mechanischer Verformung handelt, wurde von PRZIBRAM $^{1}$ erkannt, der eine große Zahl von Substanzen mit Drucken bis zu $20 \mathrm{t} / \mathrm{cm}^{2}$ preßte und anschlieBend einer $\gamma$-Bestrahlung aussetzte. Auch Bergmann und Barrington ${ }^{2}$ haben neuerdings auf einen $\mathrm{Zu}$ sammenhang zwischen Kristallstörstellen in Calcit, die durch Explosions-Schockwellen entstanden sind, und der blauvioletten Anfärbung nach Bestrahlung durch Röntgen-Licht hingewiesen. Die Autoren HaNDIN, HigGs, LewIs und WeYL ${ }^{3}$ untersuchten die Richtungsabhängigkeit der Farbzentrenbildung in gepreßten Calcit-Einkristallen. Sie beobachteten, daß die Anfärbung durch $\gamma$-Strahlung von der kristallographischen Orientierung der Kristalle während der Verformung abhängt. Bei diesen Versuchen wurden Drucke bis $2,75 \mathrm{t} / \mathrm{cm}^{2}$ angewendet.

Ein quantitativer Zusammenhang zwischen dem Ausmaß der Gitterstörungen als Folge der Verformung und der Farbzentrenkonzentration wurde von den genannten Autoren nicht angegeben. Auch über die Art der in verformten Calcitkristallen entstehenden Farbzentren ist nur sehr wenig bekannt.

Andere Farbzentren, die bei der Temperatur des flüssigen Stickstoffs durch Röntgen-Bestrahlung in unverformtem Calcit entstehen, sind durch jüngste

1 K. Przibram, Verfärbung und Lumineszenz, Springer, Berlin 1953, S. 41 u. 210.

2 O. R. Bergmann u. J. Barrington, J. Am. Ceram. Soc. 49, 502 [1966].
Arbeiten von MedLIN ${ }^{4}$ genauer bekannt. Der Absorptionsbereich dieser Farbzentren liegt im nahen Ultraviolett; sie sind mit den hier untersuchten Farbzentren nicht identisch. Cunningham ${ }^{5}$ fand nach Bestrahlung bei tiefen Temperaturen ein Absorptionsmaximum bei $650 \mathrm{~nm}$, das er dem trigonalen $\mathrm{CO}_{3}{ }^{-}$ zuordnet und das sich durch Ablösung eines Elektrons vom Carbonation bilden soll.

\section{Erzeugung und Messung von Gitterstörungen in Calcit}

Die stabile Modifikation des Calciumcarbonats, der rhomboedrische Calcit, kann durch mechanische Verformung einen ungewöhnlich hohen Störungsgrad erreichen. Die Gitterstörungen wurden für die hier beschriebenen Untersuchungen in den Kristallpulvern durch Pressen in einer Stahlmatrize oder durch Mahlen in einer Achatkugelmühle erzeugt. Das Pressen erfolgte mit Drucken von 4 bis $12 \mathrm{t} / \mathrm{cm}^{2}$. Die dabei erhaltenen Tabletten wurden für die Messungen zu einem feinen Pulver zerrieben. Das Mahlen wurde mit Benzol als Mahlflüssigkeit über Zeiträume von 5 bis 80 Stunden durchgeführt.

Oberflächenmessungen nach der BET-Methode mit Argon als Adsorbat zeigten, daß beim Mahlen die Zerkleinerung der Pulverteilchen stärker ist als beim Pressen. Das Ausgangsmaterial hatte eine Oberfläche von $0,2 \mathrm{~m}^{2} / \mathrm{g}$, beim Pressen vergrößerte sich die Oberfläche auf 1,5 bis $1,9 \mathrm{~m}^{2} / \mathrm{g}$, während die gemahlenen Proben Oberflächen zwischen 2 und 13,5 $\mathrm{m}^{2} / \mathrm{g}$ erreichten.

3 J. Handin, D. V. Higgs, D. R. Lewis u. P. K. Weyl, Bull. Geol. Soc. Am. 68, 1203 [1957].

4 W. L. Medirs, Phys. Rev. 135, 1770 [1964].

5 J. Cunningham, J. Phys. Chem. 71, 1967 [1967]. 
Die Störungen, die nach den genannten Verfahren in Calcit-Kristallpulvern erzeugt worden waren, wurden durch die Verbreiterung der Röntgen-Beugungslinien nachgewiesen und quantitativ bestimmt.

Die Breite von Röntgen-Beugungslinien ist neben der apparativ bedingten Breite im wesentlichen auf zwei Beiträge zurückzuführen, die auf Grund der unterschiedlichen Winkelabhängigkeit ihrer verbreiternden Wirkung getrennt werden können. Der eine Beitrag wird von der Dicke der kohärent streuenden Bereiche bestimmt, der andere von den Gitterverzerrungen des Kristalls. Erfolgt die Linienverbreiterung nur auf Grund kleiner Kohärenzbereiche, so gilt nach Scherrer ${ }^{6}$ die Beziehung:

$$
D=K \lambda /(\beta \cos \vartheta) .
$$

$D$ ist die mittlere Dicke der kohärent streuenden Bereiche, $\lambda$ die Wellenlänge der verwendeten Röntgen-Strahlung, $\vartheta$ der Reflexionswinkel und $\beta$ die korrigierte Breite des Röntgen-Reflexes. $K$ ist der sogenannte Formfaktor, der von der Gestalt der Kohärenzbereiche abhängt und gewöhnlich nur wenig von eins abweicht.

Erfolgt die Verbreiterung nur durch Gitterverzerrungen, so gilt nach Stokes und $\mathrm{W}_{\text {ILson }}{ }^{7}$ die Beziehung:

$$
\eta=\beta \cdot \cot \vartheta .
$$

$\eta$ ist die „scheinbare Gitterverzerrung“, sie wird in Prozent angegeben und steht im Zusammenhang mit der mittleren Abweichung der Gitterbausteine von der Ideallage in den durch Störungen verzerrten Kristallbereichen.

Im Normalfall wird die Breite der Röntgen-Beugungslinien von beiden Größen beeinflußt. Zur Trennung der beiden verbreiternden Einflüsse sind bei Auswertung der Linienbreiten Näherungsannahmen über die Verteilungsfunktionen der Verbreiterungsursachen notwendig, da diese im allgemeinen nicht bekannt sind, jedoch die Utberlagerung (Faltung) der beiden Verbreiterungsbeiträge bestimmen. Experimentelle und theoretische Untersuchungen führten dazu, daß im allgemeinen Cauchy-Funktionen oder Gauß-Funktionen als Näherungsfunktionen verwendet werden. Bei Verwendung von Cauchy-

${ }^{6}$ P. Scherrer, Gött. Nachr., Math.-Phys. Abt. 98 [1918].

7 A. R. Stokes u. A. J. C. Wilson, Proc. Phys. Soc. London 56, 174. [1944].

8 W. H. Hall, Proc. Phys. Soc. London A 62, 741 [1949].

9 P. Royen u. H. Romeis, Z. Anorg. Allg. Chem. 319, 126 [1962].
Funktionen für beide verbreiternden Einflüsse erhält man nach $\mathrm{H}_{A L L}{ }^{8}$ die Beziehung:

$$
\frac{\beta \cdot \cos \vartheta}{\lambda}=\frac{1}{D}+\frac{\eta \cdot \sin \vartheta}{\lambda} \text {. }
$$

Setzt man Gauß-Funktionen für die Verteilung der verbreiternden Größen voraus, so erhält man nach Royen und Romeis ${ }^{9}$ die Gleichung:

$$
\frac{\beta^{2} \cdot \cos ^{2} \vartheta}{\lambda^{2}}=\frac{1}{D^{2}}+\frac{\eta^{2} \cdot \sin ^{2} \vartheta}{\lambda^{2}} .
$$

Die physikalische Bedeutung der Größe $D$, die als mittlere Dicke der kohärent streuenden Bereiche bezeichnet wurde, ist problematisch, da bisher nicht geklärt ist, wodurch in einem polykristallinen Material die Kohärenz der Röntgen-Beugung effektiv unterbrochen wird. Auf jeden Fall ist die röntgenographisch ermittelte Größe $D$ immer kleiner als die Dicke der Kristallpartikel pulverförmiger Präparate. Im weiteren Text wird die Größe $D$ vereinfacht als Kristallitdicke oder Teilchengröße bezeichnet, worunter demzufolge nicht die äußerliche Dicke der Kristallteilchen zu verstehen ist.

Die Korrektur der gemessenen Integralbreiten der Röntgen-Beugungslinien erfolgte bezüglich der apparativen Verbreiterungseinflüsse nach der Korrekturfunktion von Schoening, Niekerk und HaUl ${ }^{10}$, die Abtrennung des $\alpha_{2}$-Anteils von der verwendeten $\mathrm{Cu}-\mathrm{K}_{\alpha}$-Strahlung wurde nach einer von Jones ${ }^{11}$ angegebenen Funktion durchgeführt.

Bei Anwendung der Gln. (3) und (4) macht sich eine sehr starke Anisotropie der Verbreiterungseinflüsse bei den untersuchten Calcitpräpraten bemerkbar, die eine gemeinsame Auswertung aller Reflexe erschwert. Abb. 1 zeigt diese Anisotropie bei Anwendung von Gl. (4). Bei isotroper Verteilung der Kristallitdicken und Gitterverzerrungen müßte in der gezeigten Darstellung für jedes Präparat eine Gerade resultieren, deren reziproker Ordinatenabschnitt das Quadrat der Kristallitdicke angibt und deren Steigung dem Quadrat der scheinbaren Gitterverzerrungen entspricht.

Paterson ${ }^{12}$ und Gross ${ }^{13}$, die gestörte Calcitmineralien röntgenographisch und kalorimetrisch untersuchten, führten eine getrennte Auswertung

\footnotetext{
10 F. L. R. Schoening, J. N. Van Niekerk u. R. A. W. Haul, Proc. Phys. Soc. London B 65, 528 [1952].

11 F. W. Jones, Proc. Roy. Soc. London 166 A 16 [1938].

12 M. S. Paterson, Phil. Mag. 4, 451 [1959].

13 K. A. Gross, Phil. Mag. 12, 801 [1965].
} 
einzelner Reflexe nach Gl. (1) bzw. (2) durch, ohne die beiden verbreiternden Einflüsse generell zu trennen.

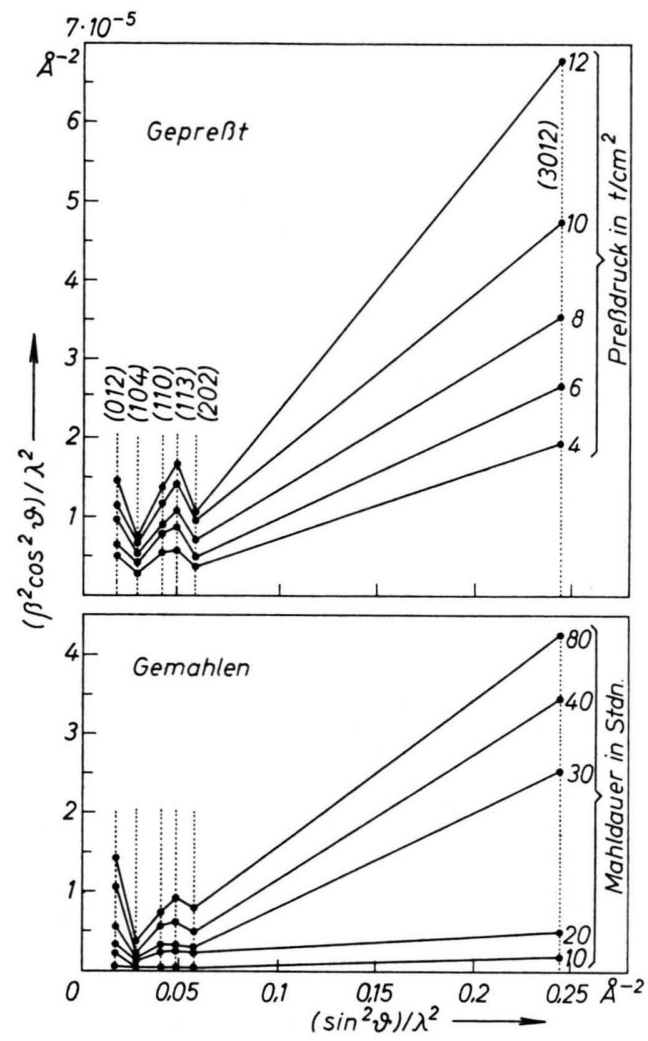

Abb. 1. Anisotropie der Integralbreiten bei den RöntgenReflexen gepreßter und gemahlener Calcitpulver.

Wie Abb. 1 zeigt, liegen die Reflexe (012) und (3012) sehr weit auseinander und sind damit für eine Bestimmung der gesuchten Größen aus diesem Reflexpaar geeignet. Die aus diesen beiden Reflexen

\begin{tabular}{|c|c|c|c|c|}
\hline Calcitpulver & $D_{\text {Cauchy }}$ & $\begin{array}{c}\eta_{\text {Cauchy }} \\
\%\end{array}$ & $\underset{\AA}{D_{\mathrm{Gau} \beta}}$ & $\underset{\%}{\eta_{\text {Gauß }}}$ \\
\hline $4 \mathrm{t} / \mathrm{cm}^{2}$ & 650 & 0,58 & 480 & 0,62 \\
\hline $6 \mathrm{t} / \mathrm{cm}^{2}$ & 610 & 0,71 & 440 & 0,88 \\
\hline $8 \mathrm{t} / \mathrm{cm}^{2}$ & 470 & 0,77 & 380 & 1,12 \\
\hline $10 \mathrm{t} / \mathrm{cm}^{2}$ & 460 & 0,96 & 360 & 1,58 \\
\hline $12 \mathrm{t} / \mathrm{cm}^{2}$ & 440 & 1,21 & 310 & 2,35 \\
\hline 5 h gem. & 1200 & 0 & 1400 & 0 \\
\hline $10 \mathrm{~h}$ gem. & 690 & 0 & 680 & 0 \\
\hline $20 \mathrm{~h}$ gem. & 740 & 0,36 & 610 & 0,29 \\
\hline $30 \mathrm{~h}$ gem. & 570 & 0,74 & 500 & 0,88 \\
\hline $40 \mathrm{~h}$ gem. & 460 & 0,83 & 350 & 1,27 \\
\hline $80 \mathrm{~h}$ gem. & 350 & 0,75 & 300 & 1,24 \\
\hline
\end{tabular}

Tab. 1. Teilchengrößen und Gitterverzerrungen gepreßter und gemahlener Calcitpulver. nach Gl. (3) und Gl. (4) ermittelten Werte für Teilchengrößen und Gitterverzerrungen der verschiedenen Calcitproben sind in Tab. 1 zusammengestellt.

Die Tabelle zeigt, daß die Kristallitdicke, die beim Ausgangsmaterial über $2000 \AA$ beträgt, schon bei dem geringsten angewendeten Preßdruck erheblich vermindert wird, dann aber bei höheren Drucken nur noch relativ wenig abnimmt (Abb. 2). Die scheinbaren Gitterverzerrungen steigen mit zunehmendem Preßdruck steil an und erreichen sehr hohe Werte (Abb. 3). Bei der Verformung durch Mahlen erfolgt eine kontinuierliche Abnahme der mittleren Teilchengrößen mit zunehmender Mahldauer (Abb. 4) und ein Anwachsen der scheinbaren Gitterverzerrungen (Abb. 5), die allerdings nicht die hohen Werte ereichen wie sie beim Pressen erhalten werden. Beide Meßgrößen nähern sich mit zunehmender Mahldauer Grenzwerten, die bei Fortsetzung des Mahlprozesses nicht mehr wesentlich verändert werden. Die Gitterstörungen scheinen sogar bei sehr langen Mahlprozessen wieder etwas zurückzugehen. Die Abb. 2 bis 5 zeigen, daß die beiden verwendeten röntgenographischen Auswertungsverfahren völlig analoge Aussagen ergeben und sich nur in den Absolutwerten unterscheiden, die bei hohen Werten der Gitterverzerrungen stark voneinander abweichen.

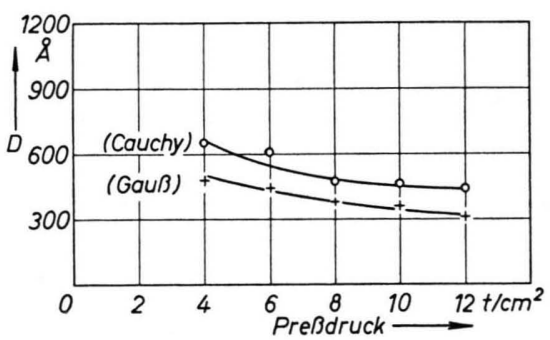

Abb. 2. Abnahme der mittleren Teilchengröße beim Pressen.

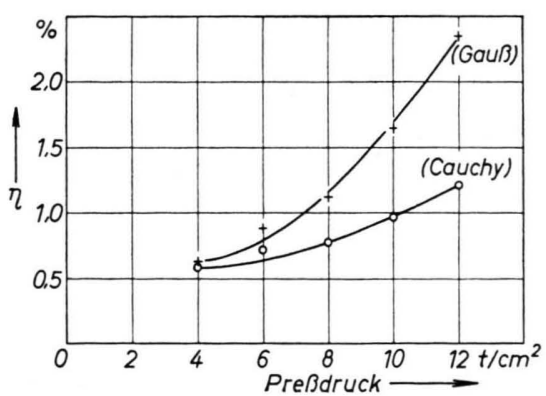

Abb. 3. Zunahme der Gitterverzerrungen beim Pressen. 


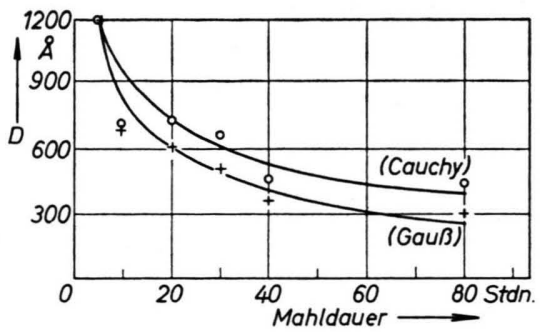

Abb. 4. Abnahme der mittleren Teilchengröße beim Mahlen.

\section{Erzeugung und Messung von Farbzentren}

Zur quantitativen Untersuchung der Farbzentrenbildung in Abhängigkeit vom Störungsgrad wurden die verformten Calcitpulver in Objektträger eingestrichen und 2,5 Stunden mit reproduzierbarer Intensität einer $\mathrm{Cu}-\mathrm{K}_{\alpha}$-Strahlung ausgesetzt. Eine längere Röntgen-Bestrahlung ergab keine weitere Vertiefung der Anfärbung. Anschließend wurden die Reflexionsspektren der bestrahlten, violett gefärbten Proben gegen einen Magnesiumoxid-Standard aufgenommen. Dabei zeigte sich, daß zwei Absorptionsmaxima auftreten. Die Maxima liegen bei 510 und $600 \mathrm{~nm}$.

Beim Vergleich der Reflexionsspektren der verformten und bestrahlten Calcitproben mit den Ergebnissen der Röntgen-Untersuchungen wird deutlich, daß ein unmittelbarer Zusammenhang zwischen Verformungsgrad und der Farbzentrenbildung besteht. Die Abb. 6 und 7 zeigen die Zunahme der beiden Absorptionsmaxima der Farbzentren mit steigendem Preßdruck bzw. mit zunehmender Mahldauer.

Aus dem gezeigten Zusammenhang kann geschlossen werden, daß die Bildung der Farbzentren an das Vorhandensein von Gitterstörungen gebunden ist, die bei der mechanischen Verformung entstehen. Ein Vergleich der Abb. 6 und 7 zeigt außerdem, daß die Farbzentrenbildung in den gepreßten Calcitproben erheblich höhere Werte annimmt als in den durch Mahlen verformten Präparaten. Nach Tab. 1 ist die Abnahme der röntgenographisch bestimmten Teilchengrößen bei beiden Arten der Verformung etwa gleich, während Gitterverzerrungen beim Pressen in erheblich größerem Ausmaß auftreten. Es ist demnach anzunehmen, daß die für die Farbzentrenbildung notwendigen Gitterstörungen entweder selbst zu röntgenographisch meßbaren Gitterverzerrungen

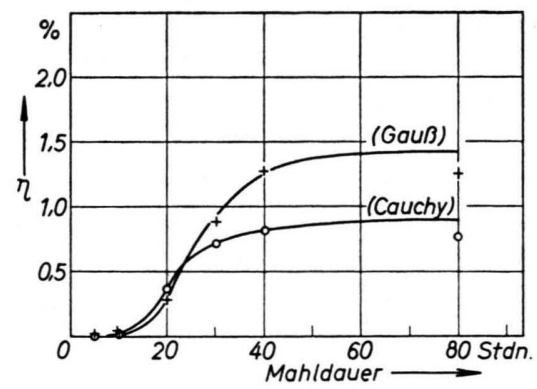

Abb. 5. Zunahme der Gitterverzerrungen beim Mahlen.

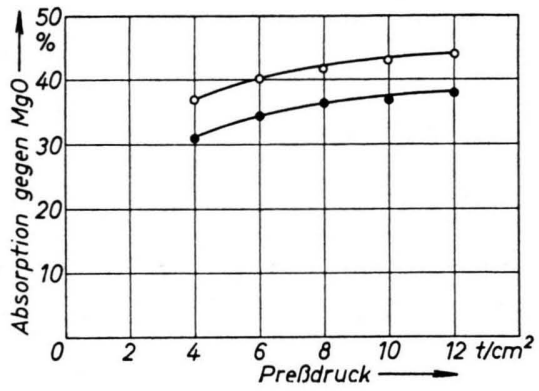

Abb. 6. Absorption gepreßter und bestrahlter Calcitpulver bei $510 \mathrm{~nm} \mathrm{(-O-O-)} \mathrm{und} 600 \mathrm{~nm}$ (- - -

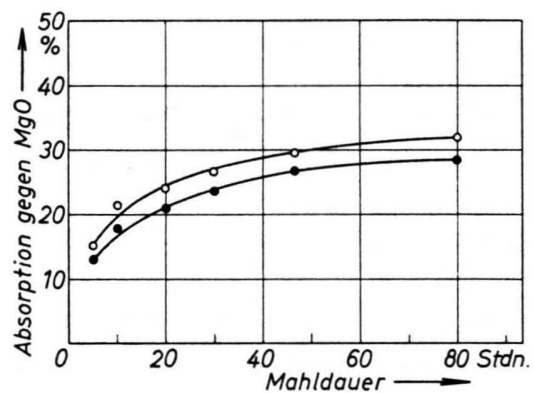

Abb. 7. Absorption gemahlener und bestrahlter Calcitpulver

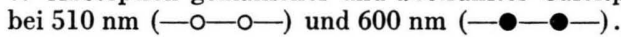

führen oder daß sie gemeinsam mit solchen Gitterstörungen entstehen. Bei sehr hohen Werten für die gemessenen Gitterverzerrungen nimmt die Farbzentrenbildung allerdings nicht mehr im gleichen $\mathrm{Ma}$ wie diese zu. Daraus kann geschlossen werden, daß nicht alle röntgenographisch als Gitterverzerrungen meßbare Störungen zur Farbzentrenbildung beitragen. Die geringe Farbzentrenbildung in verzerrungsarmen Präparaten mit geringer mittlerer Kristallitdicke und großer spezifischer Oberfläche, wie sie beim Mahlen erhalten werden, zeigt, daß Korngrenzen und Kristalloberflächen die Bildung der maßgebenden Störstellen nicht wesentlich begünstigen. 
Die röntgenographisch nachweisbaren Gitterverzerrungen, die bei der plastischen Verformung entstehen, sind zum größten Teil auf Versetzungen zurückzuführen, die bei Abgleitungsvorgängen in den Kristalliten aufgestaut werden. Diese eindimensionalen Störungen begünstigen die Entstehung nulldimensionaler Störungen im Kristallgitter, die selbst nicht wesentlich zur Verbreiterung der Röntgen-Reflexe beitragen. Die Entstehung der Farbzentren ist wahrscheinlich an das Vorhandensein bestimmter Arten solcher nulldimensionaler Fehlstellen gebunden, und somit nur indirekt mit den Breiten der Röntgen-Reflexe verknüpft. Ein direkter Einfluß nulldimensionaler Fehlstellen auf die Reflexlagen oder die Reflexintensitäten der Röntgen-Diagramme konnte bei den untersuchten Calcitproben nicht beobachtet werden.

\section{Die Erholung gestörter Calcitkristalle und ihre Wirkung auf die Farbzentrenbildung}

Werden die durch Verformung gestörten Calcitpräparate erhitzt, so erfolgt eine Abnahme der Gitterverzerrungen oberhalb bestimmter Temperaturen. Proben eines durch Pressen mit $15 \mathrm{t} / \mathrm{cm}^{2}$ stark gestörten Calcitpulvers wurden je 20 Stunden bei Temperaturen zwischen 50 und $500{ }^{\circ} \mathrm{C}$ getempert. Die Temperaturintervalle betrugen $50^{\circ}$. Eine Auswertung nach Gl. (4) ergab die in den Abb. 8 und 9 gezeigten Zusammenhänge zwischen Rekristallisationstemperatur und Gitterverzerrungen bzw. Teilchengrößen. Die Abnahme der Gitterstörungen erfolgt in zwei Stufen, von denen die erste zwischen 50 und $150{ }^{\circ} \mathrm{C}$ liegt und einer Aktivierungsenergie von etwa $90 \mathrm{kcal} / \mathrm{Mol}$ entspricht, während die restlichen Verzerrungen zwischen 250 und $500{ }^{\circ} \mathrm{C}$ mit einer Aktivierungsenergie von etwa $120 \mathrm{kcal} / \mathrm{Mol}$ ausheilen. Das Kristallwachstum erfolgt nach Abb. 9

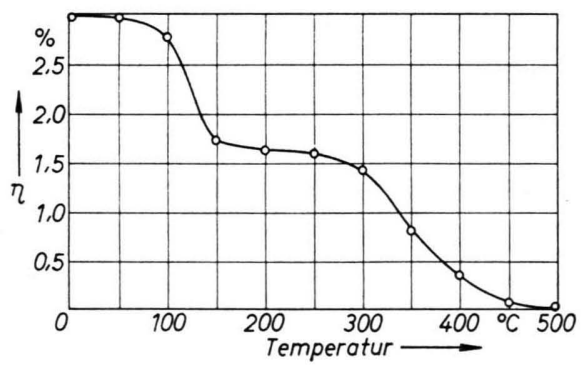

Abb. 8. Abnahme der Gitterverzerrungen nach 20 Std. Tempern. in merklichem Ausmaß erst oberhalb $200{ }^{\circ} \mathrm{C}$ und steigt dann fast linear mit der Temperatur an. Oberhalb $400{ }^{\circ} \mathrm{C}$ wurden Kristallitgrößen erhalten, die röntgenographisch nicht mehr genau zu bestimmen sind.

Die nach der Verformung bei verschiedenen Temperaturen behandelten Proben wurden zur Erzeugung von Farbzentren einer Röntgen-Bestrahlung unterzogen und die Reflexionsspektren der angefärbten Proben ausgemessen. Dabei zeigte sich, daß die Fähigkeit der Farbzentrenbildung in den getemperten Präparaten ähnlich zurückgeht wie die Gitterverzerrungen. Ein Vergleich der Abb. 8 und 10 zeigt dieses Verhalten. Die für die Farbzentrenbildung erforderlichen Störstellen heilen demnach wie die Gitterverzerrungen in zwei Stufen aus. Die erste dieser Stufen liegt im Temperaturintervall zwischen Zimmertemperatur und $150^{\circ} \mathrm{C}$, die zweite zwischen 200 und $300^{\circ} \mathrm{C}$. Die Abnahme der für die Farbzentrenbildung erforderlichen Störungen beginnt bei niedrigerer Temperatur als die Abnahme der röntgenographisch wirksamen Gitterstörungen.

Da die zu Farbzentren führenden Störungen schon bei Zimmertemperatur merklich zurückgehen, ist es erforderlich, zwischen Verformung und Farbzentrenbildung durch Röntgen-Bestrahlung die Präparate bei tiefen Temperaturen aufzubewahren.

Die Kurven in Abb. 10 lassen erkennen, daß der Rückgang der Farbzentrenbildung im unteren Temperaturbereich bis etwa $100{ }^{\circ} \mathrm{C}$ für das Absorptionsmaximum bei $510 \mathrm{~nm}$ wesentlich schneller erfolgt als für das Maximum bei $600 \mathrm{~nm}$. In einem verformten Calcit, der nicht erhitzt wurde, erhält man demnach bei der Bestrahlung Farbzentren mit einem höheren relativen Absorptionsanteil bei $510 \mathrm{~nm}$ als in einem Calcit, der nach der Verformung auf $100{ }^{\circ} \mathrm{C}$ erhitzt wurde. Bei so behandelten Präparaten ist dann nicht nur die Intensität der durch Rönt-

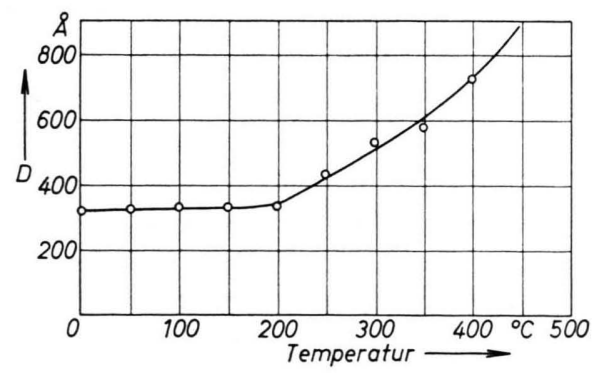

Abb. 9. Zunahme der mittleren Teilchengröße nach 20 Std. Tempern. 
gen-Strahlen erzeugten Farbzentren verschieden, sondern auch die Färbung selbst. Ein geringerer Anteil der Absorption bei $510 \mathrm{~nm}$ an der Gesamtabsorption macht sich in einer Blauverschiebung der Anfärbung bemerkbar.

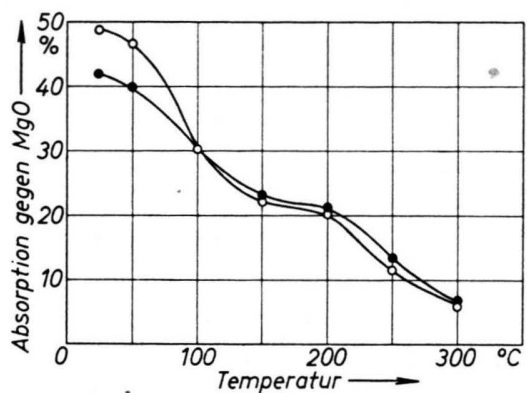

Abb. 10. Abnahme der Farbzentrenbildung nach 20 Std. Tempern. Maximum bei $600 \mathrm{~nm}$, O Maximum bei $510 \mathrm{~nm}$.

Das unterschiedliche Ansprechen der Absorptionsmaxima auf die Wärmebehandlung der Präparate kann nur erklärt werden, wenn man annimmt, daß zwei Arten von Farbzentren gebildet werden. Die Unterschiede zwischen diesen beiden FarbzentrenTypen können nicht erst bei der eigentlichen Entstehung während der Röntgen-Bestrahlung zustande kommen. Auf Grund der beschriebenen Ergebnisse muß der Unterschied auf Verschiedenheiten der Störstellen zurückgeführt werden, an denen später die Farbzentren gebildet werden. Hinweise auf die Entstehung verschiedener Farbzentren ergeben sich auch bei der Anfärbung während der Bestrahlung. Das Absorptionsmaximum bei $510 \mathrm{~nm}$ erreicht den Sättigungswert deutlich langsamer als das bei $600 \mathrm{~nm}$. Umgekehrt geht die Absorption bei $510 \mathrm{~nm}$ während des Ausbleichens der Farbzentren durch Belichtung oder Erwärmung schneller zurück als die bei $600 \mathrm{~nm}$.

Aus den in Abb. 10 gezeigten Kurven lassen sich Aktivierungsenergien für die Erholung der zu Farbzentren führenden Störstellen gewinnen. Für die erste Stufe der Erholung zwischen Zimmertemperatur und $150^{\circ} \mathrm{C}$ erhält man für die Abnahme der Absorption bei $510 \mathrm{~nm}$ eine Aktivierungsenergie von ungefähr $80 \mathrm{kcal} / \mathrm{Mol}$ und für die Absorption bei $600 \mathrm{~nm}$ etwa $70 \mathrm{kcal} / \mathrm{Mol}$. Diese Werte liegen etwas tiefer als die für die erste Abnahme der Gitterverzerrungen erhaltenen. Die zweite Stufe des Ausheilens der Störstellen kann wegen der geringen Absorptionen nicht mehr mit ausreichender Genauigkeit ausgewertet werden.
Da sich eine nach der Verformung erfolgte Tem peraturbehandlung auch auf das Verhältnis der Absorptionen der Farbzentren bei verschiedenen Wellenlängen auswirkt, während sich der Grad der Verformung nur durch eine gleichmäßige Änderung der Absorptionsintensitäten bei beiden Wellenlängen bemerkbar macht, ergibt sich die Möglichkeit die Vorgeschichte eines Calcitpräparates sowohl hinsichtlich seiner Verformung als auch bezüglich seiner Temperaturbehandlung zu untersuchen. Die röntgenographische Untersuchung liefert nur Aussagen über den Störungsgrad zum Zeitpunkt der Messung. Auf Grund einer solchen Messung kann nicht entschieden werden, ob der Störungsgrad bei der Verformung diesen Wert annahm oder ob er durch Temperaturbehandlung von einem höheren Störungsgrad auf den gemessenen Wert abgesunken ist. Nach der Erzeugung von Farbzentren durch Röntgen-Bestrahlung kann nun an Hand des Intensitätsverhältnisses der Absorptionen bei 510 und $600 \mathrm{~nm}$ auf vorausgegangene Temperaturerhöhungen zwischen 50 und $300{ }^{\circ} \mathrm{C}$ geschlossen werden.

Das Verhältnis $V$ zwischen Absorption bei $510 \mathrm{~nm}$ und Absorption bei $600 \mathrm{~nm}$ ist in Tab. 2 für die gepreßten, gemahlenen und für gepreßte und anschließend getemperte Calcitpulver zusammengestellt.

\begin{tabular}{|c|c|c|c|c|c|}
\hline $\begin{array}{l}\text { Calcit- } \\
\text { pulver }\end{array}$ & $V$ & $\begin{array}{l}\text { Calcit- } \\
\text { pulver }\end{array}$ & $V$ & $\begin{array}{l}\text { Calcit- } \\
\text { pulver }\end{array}$ & $V$ \\
\hline $\begin{array}{r}4 \mathrm{t} / \mathrm{cm}^{2} \\
6 \mathrm{t} / \mathrm{cm}^{2} \\
8 \mathrm{t} / \mathrm{cm}^{2} \\
10 \mathrm{t} / \mathrm{cm}^{2} \\
12 \mathrm{t} / \mathrm{cm}^{2}\end{array}$ & $\begin{array}{l}1,19 \\
1,17 \\
1,15 \\
1,16 \\
1,18\end{array}$ & $\begin{array}{l}5 \mathrm{~h} \text { gem. } \\
10 \mathrm{~h} \text { gem. } \\
20 \mathrm{~h} \text { gem. } \\
30 \mathrm{~h} \text { gem. } \\
40 \mathrm{~h} \text { gem. } \\
80 \mathrm{~h} \text { gem. }\end{array}$ & $\begin{array}{l}1,15 \\
1,13 \\
1,10 \\
1,13 \\
1,11 \\
1,14\end{array}$ & $\begin{array}{l}15 \mathrm{t} / \mathrm{cm}^{2} \\
50^{\circ} \text { get. } \\
100^{\circ} \text { get. } \\
150^{\circ} \text { get. } \\
200^{\circ} \text { get. } \\
250^{\circ} \text { get. } \\
300^{\circ} \text { get. }\end{array}$ & $\begin{array}{l}1,17 \\
1,16 \\
0,98 \\
0,92 \\
0,90 \\
0,89 \\
0,86\end{array}$ \\
\hline
\end{tabular}

Tab.2. Verhältnis der Absorptionsmaxima bei 510 und $600 \mathrm{~nm}$ für verformte und getemperte Calcitpräparate.

Die Tabelle zeigt, daß das Verhältnis der Absorptionen im Rahmen der Meßgenauigkeit für alle gepreßten Präparate unabhängig vom Verformungsgrad bei dem Wert 1,17 liegt. Bei den gemahlenen Präparaten beträgt der mittlere Wert 1,13 und ist ebenfalls nicht von der Mahldauer abhängig. Bei den mit $15 \mathrm{t} / \mathrm{cm}^{2}$ verformten und anschließend erwärmten Proben, die in der rechten Spalte der Tab. 2 angeführt sind, erfolgt dagegen ab $50{ }^{\circ} \mathrm{C}$ eine starke Abnahme des Verhältnisses $V$ bis zu einem Wert von 0,86 bei dem 20 Stunden auf $300^{\circ} \mathrm{C}$ erhitzten 
Präparat. Die Bestimmung der Absorptionsverhältnisse wird wegen der abnehmenden absoluten $\mathrm{Ab}$ sorption bei höher erhitzten Präparaten immer ungenauer.

Der Wert des Absorptionsverhältnisses für die gemahlenen Proben liegt mit 1,13 deutlich unter dem mittleren Wert der gepreßten Präparate. Dies ist wahrscheinlich nicht auf eine andere Verteilung der Störungen zurückzuführen, sondern auf eine stärkere lokale Erhitzung in den Calcitkristallen beim Auftreffen der Mahlkugeln. Benutzt man die in Tab. 2 gezeigte Beziehung, so müssen nach dem $\mathrm{Ab}$ sorptionsverhältnis von 1,13 während des Mahlvorganges lokale Temperaturerhöhungen auftreten, die etwas über $50{ }^{\circ} \mathrm{C}$ liegen. Auf wesentlich stärkere lokale Temperaturerhöhungen während des Mahlens ohne Mahlflüssigkeit kann man das Ausbleiben der Farbzentrenbildung in einem sehr lange trocken gemahlenen Calcitpräparat zurückführen.

Die Abhängigkeit der Absorptionsverhältnisse eines röntgenbestrahlten verformten Calcits von der thermischen Vorbehandlung könnte auch für geologische Untersuchungen von Interesse sein. Pater-
soN ${ }^{14}$ zeigte, daß in der Natur aufgefundene, verformte Calcitmineralien nicht ohne weiteres mit experimentell unter Druck verformten Proben verglichen werden können, um Aussagen über geologisch wirksam gewordene Drucke zu erhalten, da unbekannt ist, welchen Temperaturen die Mineralien nach der Verformung ausgesetzt waren. Bei geologischen Verwerfungen und Faltungen scheinen Temperaturerhöhungen der Gesteine auf mehr als $100{ }^{\circ} \mathrm{C}$ neben dem Auftreten hoher Drucke nicht selten zu sein. Wegen der hohen Störungskonzentration, die der Calcit bei plastischer Verformung erreicht, wäre er geeignet, als Indikator für geologisch wirksam gewordene Drucke zu dienen. Die genaue Kenntnis der Farbzentrenbildung bei verformtem Calcit und deren Veränderungen infolge von'Temperaturvorbehandlungen könnte eine Verwendung von Calcitmineralien für solche Untersuchungen ermöglichen.

Wir danken der Deutschen Forschungsgemeinschaft für die Bereitstellung von Forschungsmitteln.

14 M. S. Patezson, Proc. Roy. Soc. London A 271, 57 [1963]. 